\title{
Ion Range Measurements using Fluorescent Nuclear Track Detectors
}

\author{
G. Klimpki ${ }^{\mathrm{a}}$, J.-M. Osinga ${ }^{\mathrm{a}}$, R. Herrmann ${ }^{\mathrm{b}}$, M.S. Akselrod ${ }^{\mathrm{c}}$, O. Jäkel ${ }^{\mathrm{a}, \mathrm{d}, \mathrm{e}}$, S. \\ Greilich $^{\mathrm{a}}$ \\ ${ }^{a}$ German Cancer Research Center (DKFZ), Division of Medical Physics in Radiation \\ Oncology, Im Neuenheimer Feld 280, 69120 Heidelberg, Germany \\ ${ }^{b}$ Aarhus University, Department of Physics and Astronomy, Ny Munkegade 120, 8000 \\ Aarhus, Denmark \\ ${ }^{c}$ Landauer Inc., Stillwater Crystal Growth Division, 723 1/2 Eastgate, Stillwater Oklahoma \\ 74074, USA \\ ${ }^{d}$ Heidelberg Ion-Beam Therapy Center (HIT), Im Neuenheimer Feld 450, 69120 Heidelberg, \\ Germany \\ ${ }^{e}$ Heidelberg University Hospital, Department of Radiation Oncology, Im Neuenheimer Feld \\ 400, 69120 Heidelberg, Germany
}

\begin{abstract}
Fluorescent nuclear track detectors (FNTDs) show excellent detection properties for heavy charged particles and have, therefore, been investigated in this study in terms of their potential for in-vivo range measurements.

We irradiated FNTDs with protons as well as with $\mathrm{C}, \mathrm{Mg}, \mathrm{S}, \mathrm{Fe}$ and Xe ion beams $(3-6 \mathrm{MeV} / \mathrm{u})$ over a broad range of fluences $\left(4.5 \mathrm{e} 5-1.0 \mathrm{e} 11 \mathrm{~cm}^{-2}\right)$ with the detectors' optical $c$-axis positioned perpendicular to the beam direction. All measured ion ranges (for single track as well as track bulk intensity irradiations) deviate less than $3 \%$ from tabulated SRIM data, independent of particle type, energy, fluence and linear energy transfer.

Proton irradiation of detectors placed inside a polymethyl methacrylate (PMMA) phantom at the Heidelberg Ion-Beam Therapy Center showed promising results for future in-vivo FNTD applications.
\end{abstract}

Keywords: Ion range measurements, Fluorescent nuclear track detectors, Laser-induced fluorescence, Confocal laser-scanning microscopy, Heavy ion radiotherapy

\section{Introduction}

The sharp dose fall-off in proton and ion radiotherapy offers maximal dose deposition in a well-defined, narrow depth range at the distal track end. In order to fully benefit from this dose profile, precise knowledge of ion ranges in the patient is substantial, since imaging, planning and particle field composition uncertainties due to fragmentation can largely jeopardized dose conformity. Because of their superior spatial resolution, fluorescent nuclear track detectors 
(FNTDs) are promising candidates for novel in-vivo treatment plan verification tools. Implanted detectors or detectors in body cavities could help accessing direct information on a radiation treatment such as ion fluences, energies or ranges. FNTDs show excellent detection efficiency of fast neutrons and swift heavy charged particles with linear energy transfer (LET) greater than approximately $0.2 \mathrm{keV} / \mu \mathrm{m}$ (Akselrod et al., 2006). At the same time, they might serve as x-ray markers because of their high density.

FNTDs are based on single $\mathrm{Al}_{2} \mathrm{O}_{3}: \mathrm{C}, \mathrm{Mg}$ crystals. These crystals contain high concentrations of $\mathrm{F}_{2}^{2+}(2 \mathrm{Mg})$ color centers, which exhibit radiochromic transformations under ionizing radiation. Laser-induced fluorescence allows fast and non-destructive readout using confocal laser scanning microscopy (LSM). (Akselrod et al., 2011)

Irradiations of FNTDs with protons as well as with $\mathrm{C}, \mathrm{Mg}, \mathrm{S}, \mathrm{Fe}$ and Xe ions at the Max Planck Institute for Nuclear Physics in Heidelberg, Germany, and at the Accelerator Laboratory in Jyväskylä, Finland, serve as a basic feasibility study of future in-vivo FNTD applications. We measured projected ranges of these mono-energetic ion beams for various particle fluences. Measurement accuracy was analyzed using single track (low fluence) and track bulk evaluation routines (high fluence) and the influence of ion type, kinetic energy, particle fluence and LET on range precision has been investigated.

In addition, FNTDs have been irradiated at the the Heidelberg Ion-Beam Therapy Center under clinical conditions in order to simulate detector use in radiation therapy.

\section{Materials and Methods}

\subsection{Detectors}

We used single crystals of $\mathrm{Al}_{2} \mathrm{O}_{3}: \mathrm{C}, \mathrm{Mg}$ produced by Landauer Inc., which have been cut along the optical $c$-axis into small rectangular plates $(4.0 \times 6.0 \times 0.5$ $\mathrm{mm}^{3}$ ) polished on one of their large sides for optical quality (Fig. 1).

\subsection{Irradiations}

These samples have then been irradiated in vacuum with beam direction perpendicular to their edges in order to obtain full information on projected ion ranges. The entrance angle has carefully been adjusted to avoid range over- and under-estimation (Fig. 1).

The 12 MV Tandem van-de-Graaff Accelerator of the Max Planck Institute for Nuclear Physics in Heidelberg, Germany, delivered total kinetic energies of 3 (p), $6(\mathrm{p}), 48$ (C-12), $96(\mathrm{Mg}-24)$ and $100 \mathrm{MeV}$ (S-32). By changing the beam

widening with quadrupol magnets and the total irradiation time, desired particle fluences have be adjusted.

The Jyväskylä Accelerator Laboratory in Finland delivered 519 MeV Fe-56 ions and $1221 \mathrm{MeV}$ Xe-132 ions. 


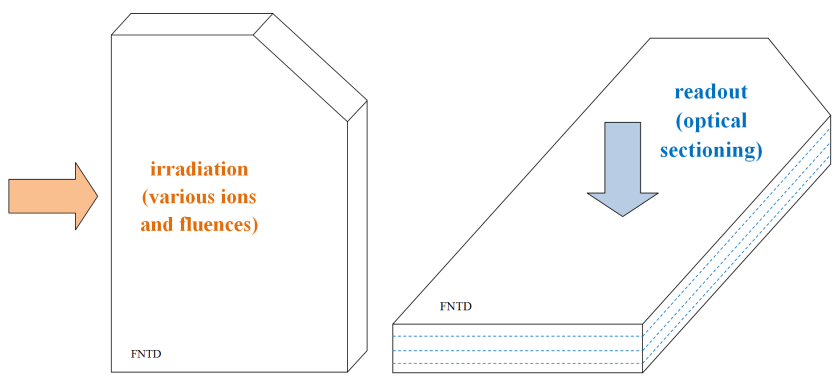

Figure 1: Schematic diagram of FNTD irradiation (left) and readout (right) based on optical sectioning under the Zeiss LSM 710 confocal microscope; polished surface on the back of the detector

\subsection{Detector readout and image processing}

The Zeiss LSM 710 ConfoCor 3 was used in the configuration described in Greilich et al., 2012, (i.e. $633 \mathrm{~nm}$ Helium-Neon laser line for excitation, avalanche photodiode with $655 \mathrm{~nm}$ longpass filter for detection) together with the 2009 ZEN control software for FNTD readout.

The 1.4 Oil DIC II, 40× objective lens provided a lateral resolution and lateral diameter of the system point spread function (PSF) of about $400 \mathrm{~nm}$. Furthermore, we chose an illumination time of $50 \mu$ s for each spot position (dwell time) and the maximum applicable laser power (appr. $100 \mu \mathrm{W}$ at sample) to avoid signal saturation. The Zeiss LSM 710 allows taking multiple images in depth by shifting the focal plane in vertical direction. Typical applied step sizes are in the order of several $\mu \mathrm{m}$. Images obtained in the same focal plane but on different lateral coordinates can be combined in a tile scan within ZEN control software. This tool was helpful when imaging a larger area of the detector edge at one defined depth. Detailed readout protocols can be found in Klimpki, 2012.

For image processing, we used ImageJ (version 1.46a) (Rasband, 2011), a public domain program that is able to import readout parameters saved by ZEN, together with customized user routines.

\section{Experiments and Results}

Fig. 2 gives an overview of carbon-irradiated FNTDs. Since irradiation covered a broad range of fluences, we established two different evaluation routines one for low $\left(\Phi<10^{7} \mathrm{~cm}^{-2}\right)$ and one for high particle fluences $\left(\Phi>10^{7} \mathrm{~cm}^{-2}\right)$. They will be referred to as single track and track bulk evaluation, respectively.

\subsection{Single track evaluation}

For fluences smaller than $10^{7} \mathrm{~cm}^{-2}$, single particle tracks were well visible under the microscope (Fig. 2, panels 1 and 2). A series of 34 images was taken in a depth interval of $100 \mu \mathrm{m}$ to ensure that track core centers lie in the focal plane of the microscope. By determining the entrance point into the detector 

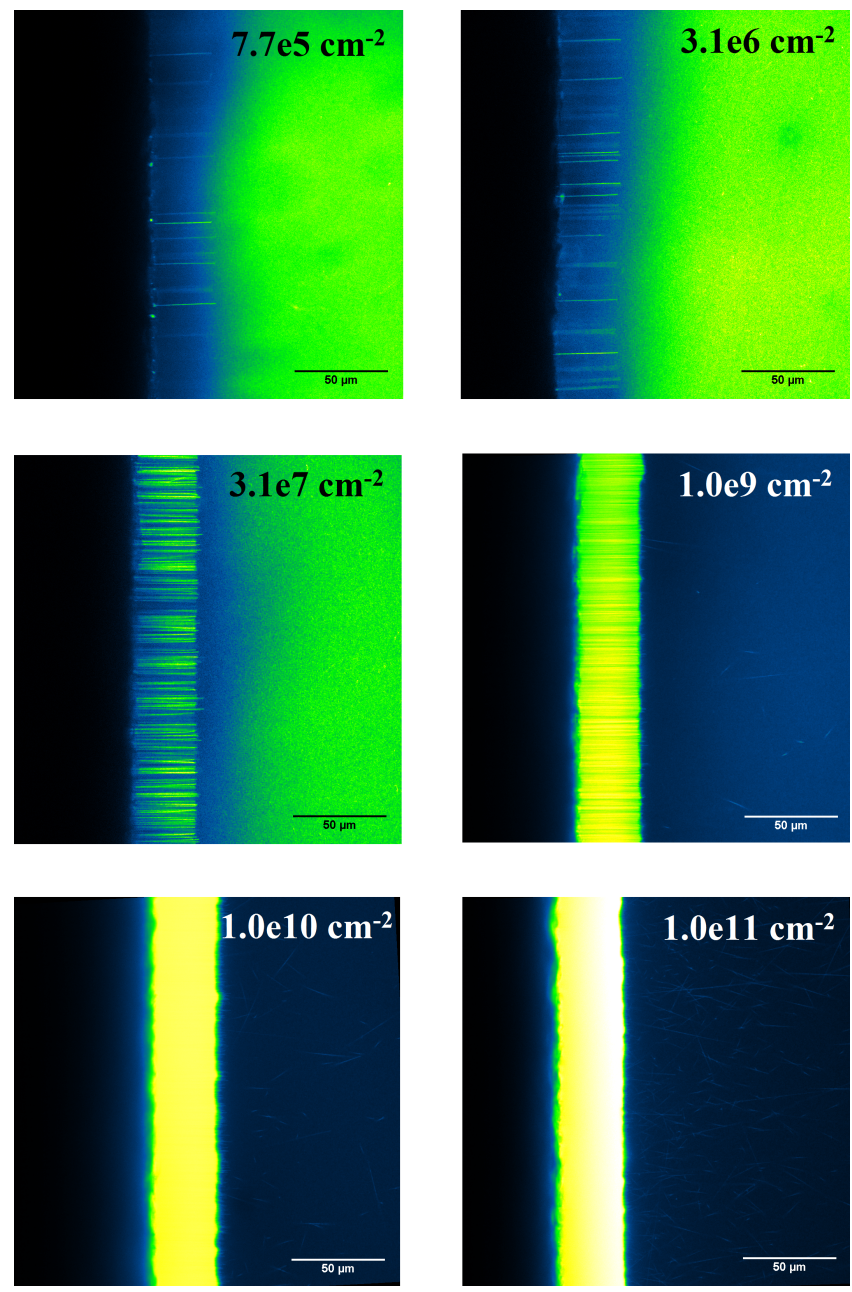

Figure 2: FNTDs irradiated with $48 \mathrm{MeV}$ C-12 ions. Carbon ions enter the detector from the left with a theoretical range tabulated by SRIM at $33.05 \mu \mathrm{m}$. The color scheme encodes fluorescence intensity (dark for low and bright for high intensity). At low fluences (i.e. top left), entrance and end point of individual tracks can be marked. The projected range was calculated from the lateral coordinates of these points. Besides, one can observe a decreased background intensity towards the detector edge. At high fluences (i.e. bottom right), single tracks can no longer be resolved. The distance between the two inflection points in the corresponding grey value profile serves as a measure for the projected range. Additional tracks representing neutrons and light fragments originating from nuclear interactions can be seen throughout the detector volume. Zeiss LSM 710 microscope settings: $100.85 \mu$ dwell time, $1 \mathrm{AU}(43.6 \mu \mathrm{m})$ pinhole diameter and maximum applicable excitation laser power. 
and the end of the particle track when the track is in focus, we could calculate the projected range. Uncertainties in the cutting of the detector edge challenge the precise identification of the ion entrance point. The results for 3,150 measured single particle tracks are given in Table 1.

In order to apply the described evaluation technique, detectors have to be irradiated perpendicular to their edge. We measured the entrance angles for a large number of particle tracks and estimated the maximum deviation due to imprecise positioning to be $\pm 5^{\circ}$.

Table 1: Range measurement results of single track evaluation

\begin{tabular}{llll}
\hline particle type & $\begin{array}{l}\text { fluence } \\
{\left[\mathrm{cm}^{-2}\right]}\end{array}$ & $\begin{array}{l}\text { measured range } \\
{[\mu \mathrm{m}]}\end{array}$ & $\begin{array}{l}\text { deviation from } \\
\text { SRIM range [\%] }\end{array}$ \\
\hline $\mathrm{Mg}-24(8+)$ & $4.5 \mathrm{e} 5$ & $24.28 \pm 0.07$ & $+(1.12 \pm 0.29)$ \\
$\mathrm{S}-32(9+)$ & $6.7 \mathrm{e} 5$ & $16.55 \pm 0.04$ & $+(1.72 \pm 0.25)$ \\
$\mathrm{C}-12(5+)$ & $7.7 \mathrm{e} 5$ & $32.77 \pm 0.08$ & $-(0.85 \pm 0.24)$ \\
protons & $\sim 1.0 \mathrm{e} 6$ & $51.24 \pm 0.39$ & $+(2.93 \pm 0.78)$ \\
protons & $\sim 1.0 \mathrm{e} 6$ & $162.9 \pm 0.5$ & $+(1.50 \pm 0.31)$ \\
Fe-56 & $\sim 1.0 \mathrm{e} 6$ & $53.11 \pm 0.25$ & $-(0.84 \pm 0.47)$ \\
$\mathrm{Xe}-132(8+)$ & $\sim 1.0 \mathrm{e} 6$ & $49.95 \pm 0.34$ & $+(1.11 \pm 0.69)$ \\
$\mathrm{C}-12(5+)$ & $3.1 \mathrm{e} 6$ & $32.59 \pm 0.04$ & $-(1.39 \pm 0.12)$ \\
\hline
\end{tabular}

\subsection{Track bulk evaluation}

For fluences greater than $10^{7} \mathrm{~cm}^{-2}$, single tracks could no longer be resolved (Fig. 2, panels $3-6$ ). Therefore, image slices of approximately $50 \mu \mathrm{m}$ height have been acquired in one distinct depth. The grey value profile of such a slice (Fig. 3) was then ascertained via ImageJ. By determining the inflection points of the curve, we could calculate the projected range. This fully automated routine has been applied to up to 250 image slices per irradiated FNTD yielding a mean range.

We chose the distance between the two inflection points to be a measure for the projected range, since an ideal grey value profile should have the shape of a rectangular function: One would expect no signal in front of the detector, an abrupt increase in fluorescence at the detector edge and an abrupt fall-off at the defined ion range back to a constant background level.

1,140 image slices have been evaluated using this fully automated track bulk evaluation routine. The results are listed in Table 2 .

\subsection{Towards in-vivo ion range measurements}

In order to further investigate the basic feasibility of FNTDs for clinical in-vivo applications, we created a therapeutic treatment plan for a polymethyl methacrylate (PMMA) cylinder containing tissue surrogates (i.e. Gammex LN450 lung, AP6 adipose, LV1 liver, CB2-30\% cortical bone) and FNTDs at the distal edge of a rectangular target volume $\left(5 \times 5 \times 1 \mathrm{~cm}^{3}\right)$. After applying $1 \mathrm{~Gy}$ protons at the Heidelberg Ion-Beam Therapy Center (multiple energy slabs for 


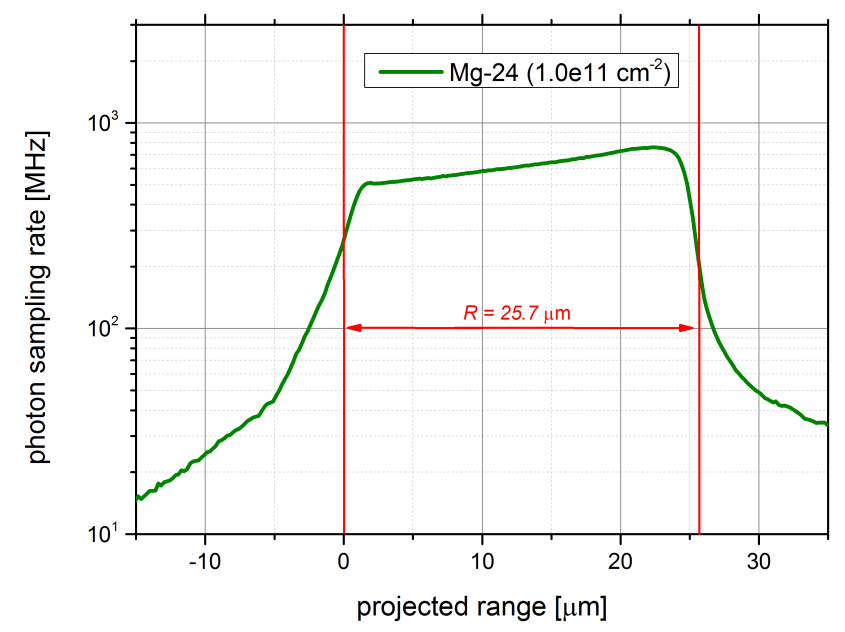

Figure 3: Grey value profile of an image slice $\left(\mathrm{Mg}-24,96 \mathrm{MeV}, 1.0 \mathrm{e} 11 \mathrm{~cm}^{-2}\right)$ with a logarithmic scale on the vertical axis. The vertical red lines represent the inflection points. Observed magnesium ions have a theoretical range tabulated by SRIM at $24.01 \mu \mathrm{m}$.

Table 2: Range measurement results of track bulk evaluation

\begin{tabular}{llll}
\hline particle type & $\begin{array}{l}\text { luence } \\
{\left[\mathrm{cm}^{-2}\right]}\end{array}$ & $\begin{array}{l}\text { measured range } \\
{[\mu \mathrm{m}]}\end{array}$ & $\begin{array}{l}\text { deviation from } \\
\text { SRIM range [\%] }\end{array}$ \\
\hline $\mathrm{C}-12(5+)$ & $3.1 \mathrm{e} 7$ & $33.13 \pm 0.06$ & $+(0.24 \pm 0.18)$ \\
$\mathrm{C}-12(5+)$ & $1.0 \mathrm{e} 8$ & $32.96 \pm 0.08$ & $-(0.27 \pm 0.24)$ \\
$\mathrm{C}-12(5+)$ & $1.0 \mathrm{e} 9$ & $33.64 \pm 0.22$ & $+(1.79 \pm 0.67)$ \\
$\mathrm{C}-12(5+)$ & $1.0 \mathrm{e} 10$ & $32.14 \pm 0.11$ & $-(2.75 \pm 0.33)$ \\
$\mathrm{C}-12(5+)$ & $1.0 \mathrm{e} 11$ & $33.23 \pm 0.07$ & $+(0.55 \pm 0.21)$ \\
$\mathrm{Mg}-24(8+)$ & $1.0 \mathrm{e} 11$ & $24.53 \pm 0.06$ & $+(0.27 \pm 0.43)$ \\
$\mathrm{S}-32(9+)$ & $1.0 \mathrm{e} 11$ & $16.31 \pm 0.07$ & $+(2.18 \pm 0.25)$ \\
\hline
\end{tabular}


homogeneous CTV irradiation), we compared the detected distal edge with the planned and forward calculated depth-dose curve (see section 4.4 and Fig. 4 for further discussion).

\section{Discussion and Outlook}

\subsection{Results of the single track evaluation}

Table 1 shows measured ranges that both over- and under-estimate the tabulated range with deviations less than $3 \%$. The single track evaluation routine was found to be time-consuming due to the individual identification of tracks.

\subsection{Results of the track bulk evaluation}

The automated fast bulk evaluation technique yielded the same level of accuracy (Tab. 2). A major influencing factor, however, is the pinhole diameter. Fluorescent light originating outside of the focal plane washes out the detector edge. We observed that the reduction of the pinhole diameter (from 1.00 to $0.31 \mathrm{AU}$ ) improved range measurement precision significantly by blocking fluorescence off focus. The overall descreas in intensity due to pinhole reduction is not an issue because of very bright fluorescence at these fluences.

\subsection{Deviation from tabulated ranges}

We believe that the most likely reason for the statistical residuals is the geometrical roughness of the detector edge with fluctuations up to $2 \mu \mathrm{m}$. A systematic dependency on energy or particle type has not been observed. The spatial resolution of FNTDs, on the other hand, cannot be the reason for the observed deviations, since structures on a submicrometer-scale can be resolved. Furthermore, radiochromic transformation of FNTD color centers is triggered by electronic interactions in the detector, but a significant influence of the linear nuclear stopping power component would cause a systematic (and additionally

smaller) shift. Finally, from an accelerator point of view, the deviation from the requested energy, the energy spread and beam stability are well below $0.1 \%$. The results of the track bulk evaluation were found to be superior to previous range results from our group (data not shown here), where FNTDs were irradiated perpendicular to their large side with $\mathrm{C}, \mathrm{Mg}$ and $\mathrm{S}$ ions of identical energy and fluence. In that case, the vertical step size of the microscope largely limited range measurement accuracy and the exact determination of the detector surface contained additional uncertainties. Final range results deviate up to $24 \%$ from SRIM tables.

\subsection{FNTDs as in-vivo radiotherapy treatment plan verification tools}

The measured intensity profile of the conducted in-vivo simulation agrees with the planned dose distribution (Fig. 4). The range measurement accuracy enabled by FNTDs (sub-micrometer scale) is much smaller than uncertainties due to a CT slice thickness of $1 \mathrm{~mm}$. Mapping the detector position with the 
forward calculated treatment plan, therefore, contained uncertainties on a submillimeter scale. Nevertheless, the therapeutic treatment plan could be verified using FNTDs and comparing detected and planned range within a fraction of a millimeter.

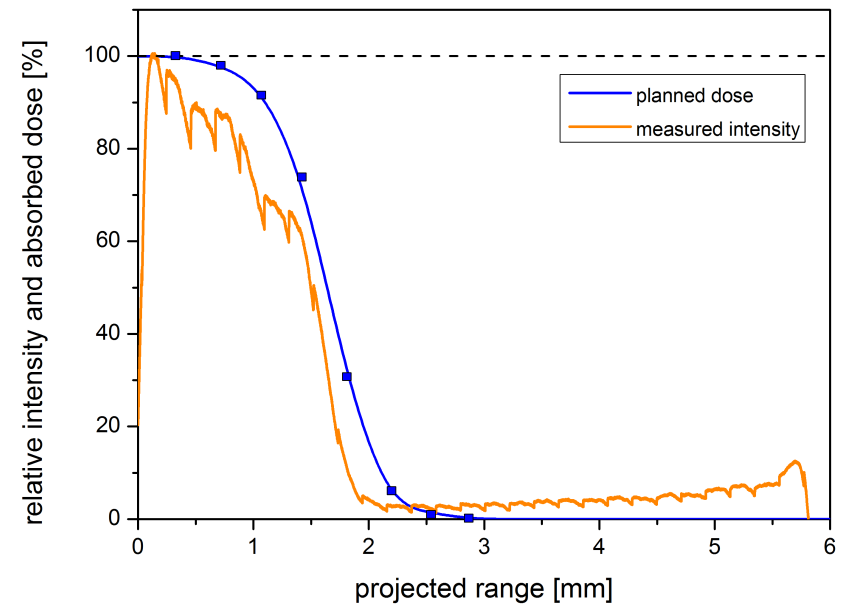

Figure 4: Comparison of planned depth-dose curve (blue) and detected intensity profile (orange). Both, measured intensity and planned dose, are normalized to their maximum. Thus, this graph allows a qualitative comparison of the two profiles. The equidistant minima seen in the curve of measured intensity originate from microscope vignetting and not color center inhomogeneities within the crystal lattice.

Another quality assurance tool could be the correlation between absorbed dose and fluorescence strength (first investigated by Sykora et al., 2008). Results of proton irradiations at the Heidelberg Ion-Beam Therapy Center suggest a defined correlation between the two quantities enabling the possibility of another direct treatment plan verification tool. However, saturation of color centers and traps within the track volume at high LETs (above $3 \mathrm{keV} / \mu \mathrm{m}$ ) (Bartz et al., 2011) might challenge this approach.

\section{Acknowledgements}

We would like to express our gratitude to Dr. Felix Bestvater from the DKFZ light microscopy facility. The authors also thank Dr. Roland Repnow and Manfred König from the Operations and Maintenance Group at the Max Planck Institute for Nuclear Physics for precise and uncomplicated FNTD irradiation as well as Dr. Michael Holzscheiter for putting us into contact with them. Furthermore, we appreciate the technical advisory and support during irradiations and treatment planning offered by Dr. Stephan Brons, Dr. Peter Heeg, Benjamin Ackermann and Swantje Ecker from HIT. This work was supported by the Helmholtz Association (translating hadron therapy from basic research to clinical application, VH-VI-303, S.G.). 


\section{References}

Akselrod, G.M., Akselrod, M.S., Benton, E.R., Yasuda, N., 2006. A novel $\mathrm{Al}_{2} \mathrm{O}_{3}$ fluorescent nuclear track detector for heavy charged particles and neutrons. Nucl. Instr. Meth. B 247, 295-306.

Akselrod, M.S., Sykora, G.J., 2011. Fluorescent nuclear track detector technology - a new way to do passive solid state dosimetry. Radiat. Meas. 46, $1671-1679$.

Bartz, J.A., Sykora, G.J., Underwood, T.H., Nichiporov, D.N., Sawakuchi, G.O., Akselrod, M.S., 2011. Evaluation of aluminum oxide fluorescent and OSL detectors in proton radiotherapy beams. Radiat. Meas. 46, 1974-1978.

Greilich, S., Osinga, J.-M., Lauer, F.M., Niklas, M., Klimpki, G., Sellner, S., Bartz, J.A., Akselrod, M.S., Jäkel, O., 2012. Fluorescent nuclear track detectors as a tool for ion-beam therapy research. these proceedings

Klimpki, G., 2012. Towards in-vivo ion range measurements using fluorescent nuclear track detectors. Bachelor thesis at DKFZ. URL (persistent): http://www.ub.uni-heidelberg.de/archiv/13839.

Rasband, W., 2011. ImageJ (version 1.46a). URL: http://rsbweb. nih.gov/ij/.

Sykora, G.J., Salasky, M., Akselrod, M.S., 2008. Properties of novel fluorescent nuclear track detectors for use in passive neutron dosimetry. Radiat. Meas. 43, 1017-1023.

Ziegler, J.F., Biersack, J.P., Ziegler, M.D., 2009. SRIM - The stopping and range of ions in matter. Lulu Press Inc., Raleigh, NC. 